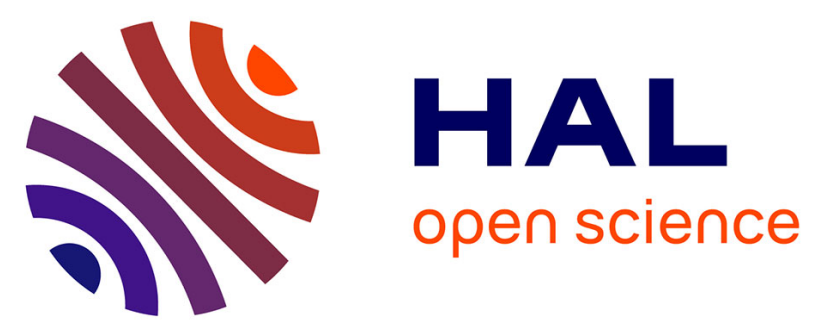

\title{
Étude des niveaux fortement excités du 12C par la polarisation des protons diffusés inélastiquement
}

X. de Bouard, J.G. Fox, B. Geoffrion, Nicolas C.M. Marty, C. Rolland, B. Tatischeff

\section{- To cite this version:}

X. de Bouard, J.G. Fox, B. Geoffrion, Nicolas C.M. Marty, C. Rolland, et al.. Étude des niveaux fortement excités du 12C par la polarisation des protons diffusés inélastiquement. Journal de Physique, 1963, 24 (11), pp.1059-1062. 10.1051/jphys:0196300240110105900 . jpa-00205602

\section{HAL Id: jpa-00205602 https://hal.science/jpa-00205602}

Submitted on 1 Jan 1963

HAL is a multi-disciplinary open access archive for the deposit and dissemination of scientific research documents, whether they are published or not. The documents may come from teaching and research institutions in France or abroad, or from public or private research centers.
L'archive ouverte pluridisciplinaire HAL, est destinée au dépôt et à la diffusion de documents scientifiques de niveau recherche, publiés ou non, émanant des établissements d'enseignement et de recherche français ou étrangers, des laboratoires publics ou privés. 


\title{
ETUDE DES NIVEAUX FORTEMENT EXCITÉS DU ${ }^{12} \mathrm{C}$ PAR LA POLARISATION DES PROTONS DIFFUSÉS INÉLASTIQUEMENT
}

\author{
Par X. DE BOUARD, J. G. FOX, B. GEOFFRION, N. MARTY, \\ C. ROLLAND et B. TATISCHEFF,
}

\begin{abstract}
Résumé. - On compare, dans le cas de niveaux bien connus, les valeurs expérimentales de polarisation à celles prévues dans l'approximation d'impulsion. En accord général avec la théorie, à l'exception des niveaux à parité favorisée avec $\Delta T=0$, les polarisations sont faibles. Les niveaux pour lesquels les polarisations sont élevées peuvent donc être considérés pour ${ }^{12} \mathrm{G}$ comme $T=0$ parité favorisée.
\end{abstract}

Abstract. - In general agreement with theory, protons inelastically scattered in the excitation of levels other than $\Delta T=0$ favoured parity, show a very low polarization. Polarization of $150 \mathrm{MeV}$ protons from excited states of high energy in $\mathrm{C}^{12}$ gives information on the nature of these levels.

Plusieurs études ont porté, ces dernières années sur la diffusion inélastique des protons d'énergie moyenne (100 à $200 \mathrm{MeV})$ sur des noyaux légers. Si dans le cas de niveaux d'énergie faible la forme de la section efficace différentielle permet d'apporter des renseignements sur la nature de l'état excité, pour les niveaux très éloignés du fondamental ceci ne semble pas le cas.

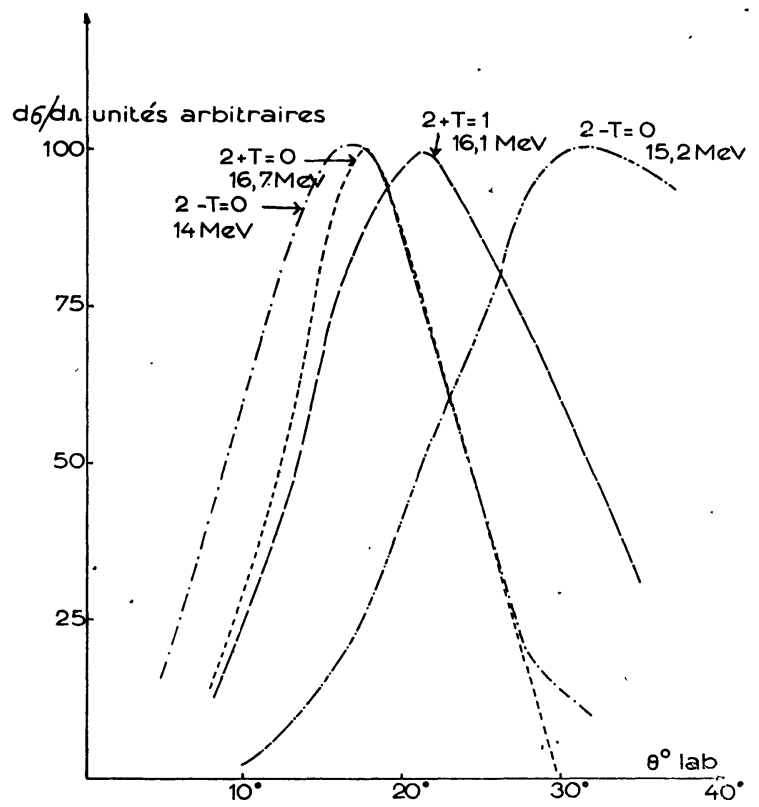

FIG. 1. - Prévisions théoriques pour certains niveaux excités de ${ }^{12} \mathrm{C}$ au voisinage de $16 \mathrm{MeV}$ (N. Vinh Mau, thèse).

Figure 1 sont données, ramenées à la même valeur maximum, les sections efficaces différentielles calculées par N. Vinh Mau [1] dans le modèle à particules indépendantes avec interaction trou-particule, pour des niveaux de ${ }^{12} \mathrm{C}$ d'énergie voisine de $16 \mathrm{MeV}$; on y observe une différence plus grande entre deux niveaux de même nature $(2-T=0)$ qu'entre les niveaux différents $(2+T=0$ et $2-T=0)$.

Nous avons cherché à voir si la mesure de la polarisation des protons diffusés inélastiquement permettait d'apporter des renseignements complémentaires sur la nature des niveaux d'énergie élevée.

Utilisant la matrice de diffusion nucléon-nucléon et l'approximation d'impulsion, Kerman et al. [2] ont montré que la polarisation des protons diffusés inélastiquement dépend des états initial et final du moment angulaire et du spin isotopique du noyau cible. Pour des angles inférieurs à $40^{\circ}$, on ne doit s'attendre à des polarisations élevées (supérieures à $50 \%$ ) que pour des transitions à parité favorisée sans changement de spin isotopique. Les résultats expérimentaux obtenus à Uppsala [3] confirment ces prévisions, surtout pour le cas des niveaux à polarisation élevée (niveaux en général d'excitation peu élevée).

Nous avons repris en détail les mesures de polarisation pour les niveaux fortement excités de ${ }^{12} \mathrm{C}$, en mesurant d'abord la polarisation des niveaux de nature connue $(15,1 \mathrm{MeV} 1+T=1,22,3 \mathrm{MeV}$ $1-T=1$ ) pour vérifier avec plus de précision les prévisions théoriques dans le cas des niveaux autres que ceux à parité favorisée $\Delta T=0$.

Les protons de $155 \mathrm{MeV}$ du synchrocyclotron d'Orsay, diffusés par une cible de graphite, correspondant à une perte d'énergie de $8 \mathrm{MeV}$, étaient analysés en énergie à l'aide d'un analyseur magnétique. La résolution en énergie du faisceau diffusé inélastiquement était d'environ $1,4 \mathrm{MeV}$, en raison de la largeur en énergie du faisceau incident et des dimensions de la cible. La polarisation des protons diffusés était alors mesurée à l'aide d'un analyseur de polarisation par diffusion sur cible de carbone 


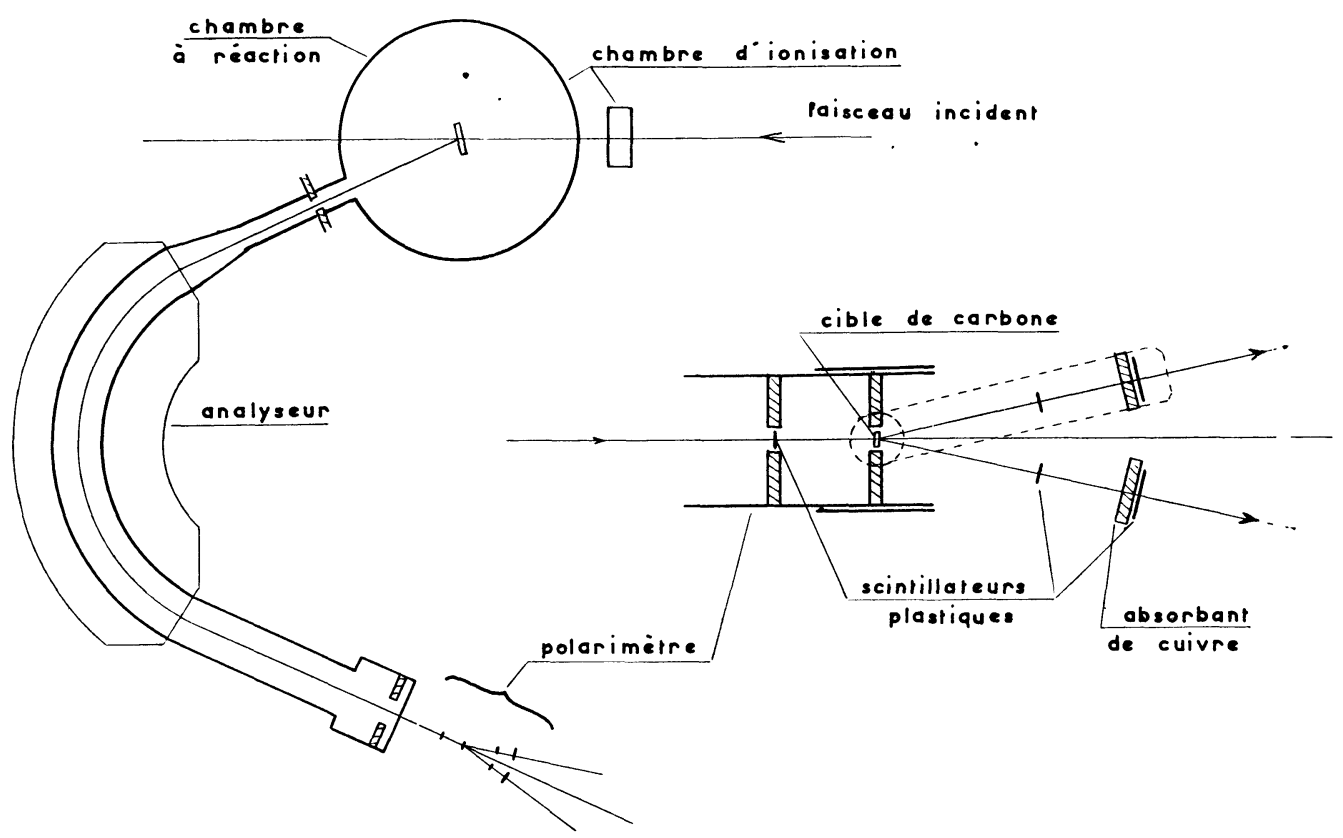

FIG. 2. - Dispositif expérimental.

comprenant un premier plastique situé avant la cible et deux télescopes à deux scintillateurs chacun, interchangeables par rotation autour de l'axe du faisceau diffusé qui est aussi l'axe du cylindre supportant ces télescopes (fig. 2). Le pouvoir d'analyse du polarimètre avait été mesuré au préalable pour différentes énergies des protons entre 125 et $150 \mathrm{MeV}$. Ce polarimètre sera décrit en détail dans une publication ultérieure, ainsi que la méthode d'analyse des résultats expérimentaux. En effet, en raison du grand pouvoir dispersif de l'analyseur magnétique d'une part et du nombre de niveaux voisins d'autre part, la détermination de la polarisation pour un niveau ne peut être faite qu'en tenant compte de la répartition en intensité, sur la cible du polarimètre, des protons diffusés par les différents niveaux ainsi que de la polarisation de chacun des niveaux. Les calculs ont été faits simultanément pour l'ensemble des niveaux à l'aide d'une IBM 650.

Les résultats expérimentaux sont peu précis en raison de la difficulté de décomposer des raies nombreuses et voisines. Nous avons pu séparer, entre 15 et $24 \mathrm{MeV}$, les principaux niveaux suivants : 15,1, $16,2,18,2,19,7,21$ et $22,3 \mathrm{MeV}$, en accord avec Jacmart et al. [4]. D'autre part, dans ce domaine d'énergie existe un fond continu de protons pour lesquels on a supposé que la polarisation variait linéairement entre les valeurs d'énergie où on peut la mesurer.

Les résultats expérimentaux pour la polarisation des protons du niveau de 15,1 MeV (fig. 3) donnent des valeurs positives et faibles en accord général avec les prévisions théoriques. Cependant ces

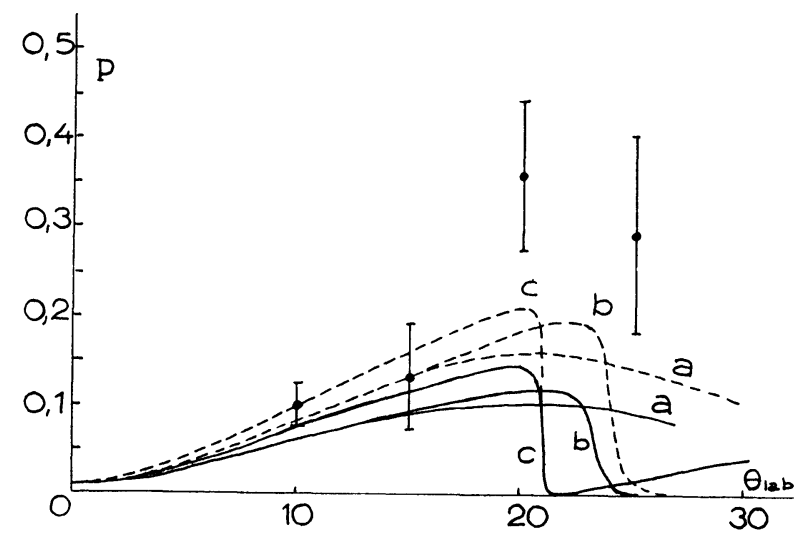

Fig. 3. - Niveau de 15,1 $\mathrm{MeV}, 1+T=1$, du ${ }^{12} \mathrm{G}$.

- _ - _ - Gammel-Thaler.

Breit et al. YLAM.

a : onde $T \doteq 0$ seule.

YLAN 3M.

$\mathrm{b}:$ ondes planes.

$\mathrm{c}:$ ondes distordues.

valeurs sont plus élevées que celles calculées à partir des paramètres de phase de la diffusion nucléon-nucléon donnés soit par le potentiel Gammel-Thaler, soit par les solutions YLAM pour $T=1$ et YLAN 3 et YLAN $3 \mathrm{M}$ pour $T=0$ de Breit et col. L'écart est important aux angles de diffusion les plus grands où les phénomènes de distorsion deviennent importants. Ceux-ci ont été calculés en utilisant un potentiel optique purement imaginaire sans interaction de spin [5].

Nous avons représenté (fig. 4) les sections effi- 

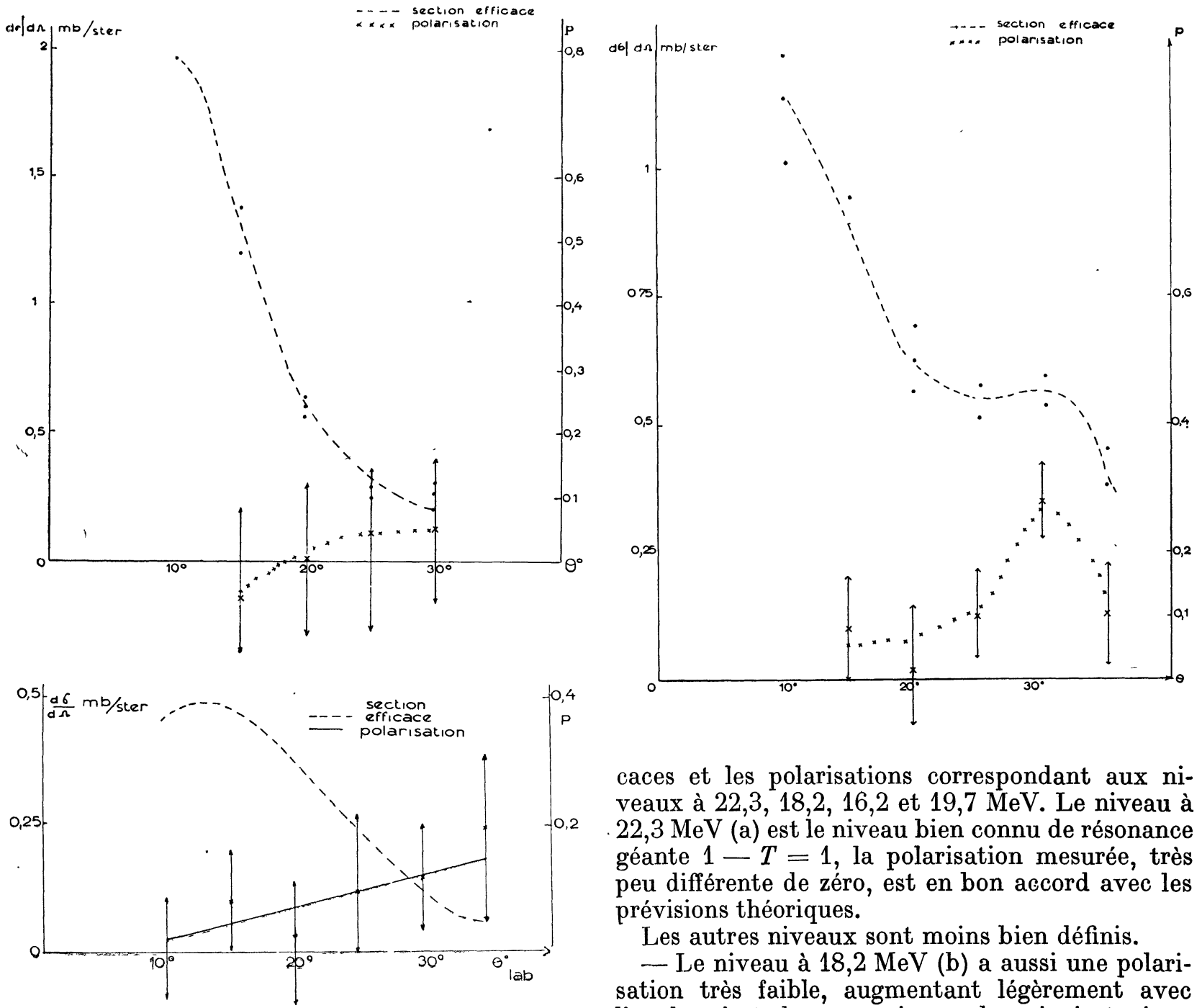

caces et les polarisations correspondant aux niveaux à $22,3,18,2,16,2$ et $19,7 \mathrm{MeV}$. Le niveau à $22,3 \mathrm{MeV}$ (a) est le niveau bien connu de résonance géante $1-T=1$, la polarisation mesurée, très peu différente de zéro, est en bon accord avec les prévisions théoriques.

Les autres niveaux sont moins bien définis.

- Le niveau à 18,2 MeV (b) a aussi une polarisation très faible, augmentant légèrement avec l'angle, c'est donc un niveau de spin isotopique

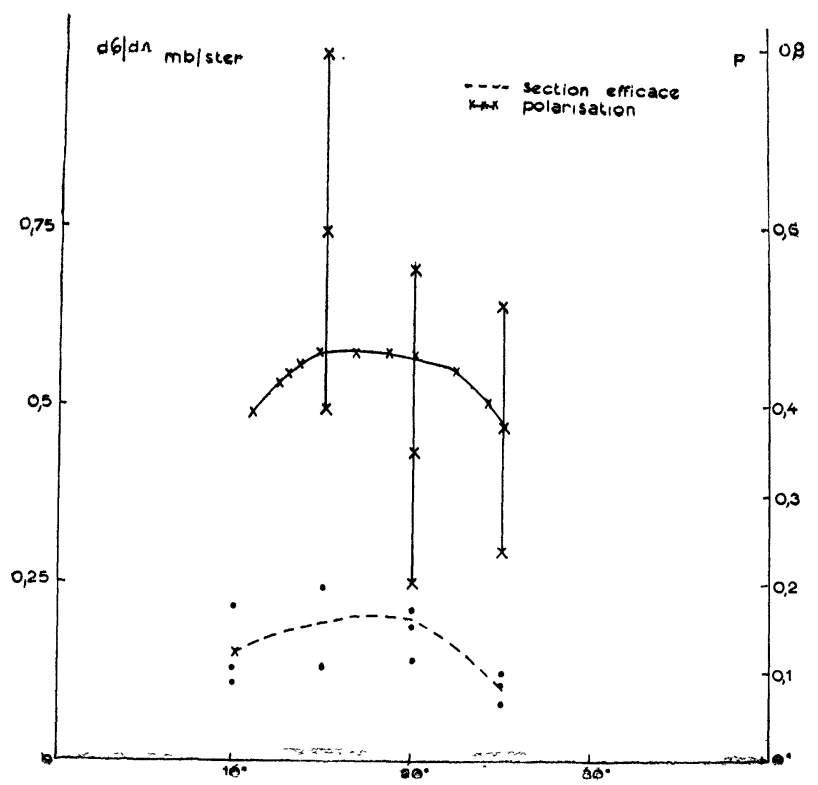
$T=1$ ou $T=0$ à parité non favorisée.

- Le niveau à 16,2 MeV (c) montre une polarisation qui, sans atteindre celle des niveaux connus $\Delta T=0$ à parité favorisée, est plus élevée que dans les autres cas que nous avons étudiés jusqu'ici; nous supposons que c'est, soit un niveau $T=0$ à parité

\begin{tabular}{|c|c|c|c|c|}
\hline \multicolumn{3}{|c|}{ Niveau prévu } & & \multirow[t]{2}{*}{ RÉSULTAT EXPÉRIMENTAL } \\
\hline$E$ en $\mathrm{MeV}$ & $J$ & $\pi$ & $T$ & \\
\hline $17 \overline{4}$ & $\overline{1}$ & $\bar{t}$ & $\overline{1}$ & $15 \overline{1} \mathrm{MeV}$ \\
\hline 23,7 & 1 & - & 1 & $22,3 \mathrm{MeV}$ \\
\hline 20,6 & 2 & 一 & 0 & $18,2 \mathrm{MeV}$ \\
\hline 19,8 & 2 & - & 1 & $19,7 \mathrm{MeV}$ \\
\hline 18,75 & 3 & - & 0 & apparaît sous le 19,7 à $30^{\circ}$ \\
\hline 16,7 & 2 & + & 0 & $162 \mathrm{MeV}$ \\
\hline 16,07 & 2 & + & 1 & $16,2 \mathrm{MeV}$ \\
\hline $\left.\begin{array}{l}14 . \\
15,2\end{array}\right\}$ & 2 & 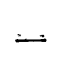 & 0 & $\begin{array}{l}\text { contribuent au fond peu polarisé } \\
\text { qui apparait aux angles }>25^{\circ}\end{array}$ \\
\hline
\end{tabular}


favorisée où le spin flip serait important, soit plutôt la superposition de deux niveaux voisins dont l'un est $T=0(-1)$.

- Enfin le niveau à 19,7 MeV (d) présente une polarisation faible, sauf à $30^{\circ}$, angle où la courbe de section efficace indique la présence d'un autre niveau, auquel on serait alors conduit à attribuer une polarisation élevée.

En utilisant les résultats apportés par les mesures de section efficace et de polarisation, nous avons essayé d'attribuer des valeurs de spin, parité, spin isotopique aux niveaux excités mis en évidence et de les interpréter par comparaison avec les principaux niveaux prévus théoriquement entre 14 et $25 \mathrm{MeV} \cdot[1]$.

Conclusion. - Les mesures de polarisation des protons diffusés inélastiquement permettent donc de mettre en évidence les transitions $\Delta T=0$ à parité favorisée.

\section{BIBLIOGRAPHIE}

[1] Vinh Mau (N.), Thèse, Annales de Physique, 1963.

[2] Kerman (A. K.), MaManus (H.) et Thaler (R. M.), Ann. Physics, 1959, 8, 475.

[3] Hillman (P.), Johansson (A.) et Tibell (G.), Nucl. Physics, 1957, 4, 648.

Alphonce (R.), Johansson (A.) et Tibell (G.), $\mathrm{Nucl}$. Physics, 1957, 4, 672.
Tyren (H.), Hillman (P.) et Johansson (A.), Nucl. Physics, 1957, 3, 336.

Johansson (A.), Tibell (G.) et Hillman (P.), Nucl. Physics, 1959, 11, 540.

[4] Jacmart (J. G.), Garron (J. P.), Riou (M.) et Ruhla (C.), International symposium on direct interactions, Padoue, 1962 (à paraître).

[5] Loncke (Ph.), Physics Letters (à paraître). 